\title{
Finite-Difference Beam Propagation Method Based on the Generalized Douglas Scheme for a Nonuniform Grid
}

Junji Yamauchi, Member, IEEE, Jun Shibayama, Minoru Sekiguchi, and Hisamatsu Nakano, Fellow, IEEE

\begin{abstract}
The finite-difference beam propagation method based on the Douglas scheme is extended to a nonuniform grid. The truncation error is reduced to $O(\Delta x)^{4}$, provided the grid growth factor is $r=1+O(\Delta x)$, while maintaining a tridiagonal matrix. The numerical results show that the accuracy is improved as compared with that obtained from the conventional nonuniform Crank-Nicholson scheme.
\end{abstract}

Index Terms - Finite-difference methods, optical propagation, optical waveguides.

\section{INTRODUCTION}

$\mathbf{T}$ $O$ IMPROVE computational efficiency of the finitedifference beam propagation method (FD-BPM), a nonuniform transverse sampling grid is often employed [1]-[5]. The truncation error of the conventional nonuniform difference approximation to the second derivative is $O(\Delta x)^{2}$, provided the grid growth factor is $r=1+O(\Delta x)$. Yevick et al. [1] refined the conventional nonuniform operator of the second derivative and improved its accuracy. Unfortunately, this results in a pentadiagonal matrix with time-consuming procedures.

For a uniform grid, the truncation error can be reduced to $O(\Delta x)^{4}$ by using the Douglas scheme, maintaining a tridiagonal matrix [6]. Recently, the authors have also developed the modified FD-BPM based on the generalized Douglas (GD) scheme for variable coefficients [7]. However, it has been believed that the Douglas scheme is valid only for a uniform grid [6]. In 1995, the authors pointed out that Douglas scheme can be extended to a nonuniform grid [8].

In this letter, we present the detailed derivation of a novel FD-BPM based on the Douglas scheme generalized for a nonuniform grid. The philosophy behind the present formulation is to eliminate the low-order error terms in Taylor series expansion. It is demonstrated that the truncation error can be reduced to $O(\Delta x)^{4}$, provided the grid growth factor is $r=1+O(\Delta x)$. For the assessment of the accuracy, the effective index of a graded-index waveguide is calculated using the imaginary-distance BPM [9]-[11]. In addition, the overlap integral between the numerically determined field and the exact solution is evaluated. It is found that the present scheme offers significant improvement, as compared with the conventional nonuniform Crank-Nicholson (CN) scheme.

Manuscript received June 27, 1996; revised September 9, 1996

The authors are with the College of Engineering, Hosei University, Koganei, Tokyo 184, Japan.

Publisher Item Identifier S 1041-1135(97)00550-8.

\section{FORMULATION}

We derive a high-accuracy nonuniform scheme in a twodimensional (2-D) problem. The Fresnel equation is expressed as

$$
\sigma \frac{\partial E}{\partial z}=\frac{\partial^{2} E}{\partial x^{2}}+\nu E
$$

where $\sigma=2 j k n_{0}$ and $\nu=k^{2}\left[n^{2}(x, z)-n_{0}^{2}\right]$ in which $k$ is the free space wavenumber, $n(x, z)$ is the index profile of the waveguide, and $n_{0}$ is the reference index to be appropriately chosen.

The nonuniform sampling grid under consideration is shown in the inset of Fig. 2, where $r$ represents the grid growth factor. By Taylor series expansion, $E_{i+1}$ and $E_{i-1}$ are expressed as

$$
\begin{aligned}
E_{i+1}=E_{i} & +r \Delta x \frac{\partial E_{i}}{\partial x}+\frac{1}{2} r^{2} \Delta x^{2} \frac{\partial^{2} E_{i}}{\partial x^{2}} \\
& +\frac{1}{6} r^{3} \Delta x^{3} \frac{\partial^{3} E_{i}}{\partial x^{3}}+\frac{1}{24} r^{4} \Delta x^{4} \frac{\partial^{4} E_{i}}{\partial x^{4}} \\
E_{i-1}= & E_{i}-\Delta x \frac{\partial E_{i}}{\partial x}+\frac{1}{2} \Delta x^{2} \frac{\partial^{2} E_{i}}{\partial x^{2}} \\
& -\frac{1}{6} \Delta x^{3} \frac{\partial^{3} E_{i}}{\partial x^{3}}+\frac{1}{24} \Delta x^{4} \frac{\partial^{4} E_{i}}{\partial x^{4}}
\end{aligned}
$$

From (2) and (3), we have

$$
\begin{aligned}
\frac{\partial^{2} E_{i}}{\partial x^{2}}= & \frac{2}{\left(r^{2}+1\right) \Delta x^{2}} \\
& \cdot\left[E_{i+1}-2 E_{i}+E_{i-1}-(r-1) \Delta x \frac{\partial E_{i}}{\partial x}\right. \\
& \left.-\frac{1}{6}\left(r^{3}-1\right) \Delta x^{3} \frac{\partial^{3} E_{i}}{\partial x^{3}}-\frac{1}{24}\left(r^{4}+1\right) \Delta x^{4} \frac{\partial^{4} E_{i}}{\partial x^{4}}\right] \\
\frac{\partial E_{i}}{\partial x}= & \frac{\delta E_{i}}{\Delta x}-\frac{1}{2}(r-1) \Delta x \frac{\partial^{2} E_{i}}{\partial x^{2}}-\frac{1}{6}\left(r^{2}-r+1\right) \Delta x^{2} \frac{\partial^{3} E_{i}}{\partial x^{3}} \\
& -\frac{1}{24}\left(r^{2}+1\right)(r-1) \Delta x^{3} \frac{\partial^{4} E_{i}}{\partial x^{4}}
\end{aligned}
$$

where

$$
\delta E_{i}=\frac{E_{i+1}-E_{i-1}}{r+1} .
$$

By substituting (5) into (4), the second derivative can be written in the form

$$
\begin{aligned}
\frac{\partial^{2} E_{i}}{\partial x^{2}}= & \frac{\delta^{2} E_{i}}{\Delta x^{2}}-\frac{1}{3}(r-1) \Delta x \frac{\partial^{3} E_{i}}{\partial x^{3}} \\
& -\frac{1}{12}\left(r^{2}-r+1\right) \Delta x^{2} \frac{\partial^{4} E_{i}}{\partial x^{4}}
\end{aligned}
$$




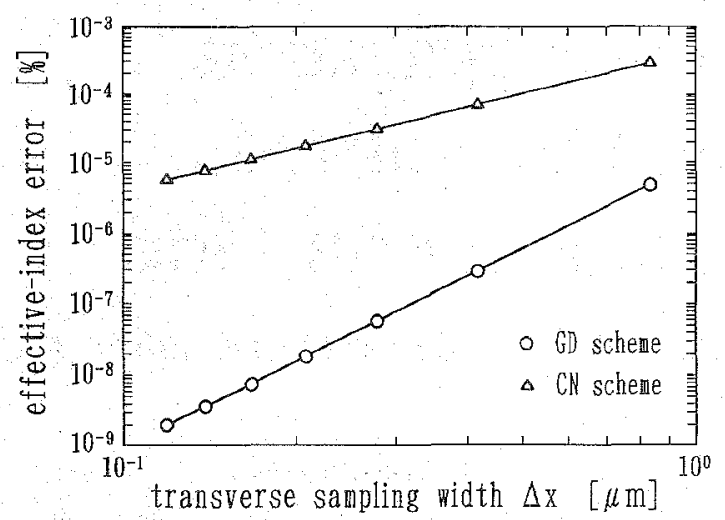

Fig. 1. Effective-index error as a function of transverse sampling width $(r=1)$.

where

$$
\delta^{2} E_{i}=\frac{2}{r+1}\left[E_{i-1}-(1+1 / r) E_{i}+E_{i+1} / r\right]
$$

Evidently, the first terms in the right-hand sides of (5) and (7) are the conventional nonuniform approximations to the first and second derivatives, respectively. Equation (8) has widely been used to analyze an optical waveguide [2]-[4]. It should be noted that (6) and (8) have the truncation error of $O(\Delta x)^{2}$ under the condition of $r=1+O(\Delta x)$.

We now introduce the GD scheme [7] in order to evaluate the third and fourth derivatives in (7). Using (1), these derivatives are rewritten as

$$
\begin{aligned}
& \frac{\partial^{3} E_{i}}{\partial x^{3}}=\frac{\delta}{\Delta x}\left(\sigma \frac{\partial E}{\partial z}-\nu E\right)_{i} \\
& \frac{\partial^{4} E_{i}}{\partial x^{4}}=\frac{\delta^{2}}{\Delta x^{2}}\left(\sigma \frac{\partial E}{\partial z}-\nu E\right)_{i}
\end{aligned}
$$

It should be noted that the truncation error can be reduced to $O(\Delta x)^{4}$, provided the condition of $r=1+O(\Delta x)$ is satisfied.

From the foregoing, we can establish

$$
\begin{aligned}
\frac{\delta^{2} E_{i}}{\Delta x^{2}}= & \frac{3 r^{2}-3 r+1}{6 r(r+1)}\left(\sigma \frac{\partial E}{\partial z}-\nu E\right)_{i+1} \\
& +\frac{-r^{2}+7 r-1}{6 r}\left(\sigma \frac{\partial E}{\partial z}-\nu E\right)_{i} \\
& +\frac{r^{2}-3 r+3}{6(r+1)}\left(\sigma \frac{\partial E}{\partial z}-\nu E\right)_{i-1}
\end{aligned}
$$

Alternatively, we can replace $\delta$ in (9) by the following three-point representation:

$$
\delta E_{i}=\frac{\left(E_{i+1}-E_{i}\right) / r+r\left(E_{i}-E_{i-1}\right)}{r+1} .
$$

In this case, we obtain the slightly different result:

$$
\begin{aligned}
\frac{\delta^{2} E_{i}}{\Delta x^{2}}= & \frac{r^{2}+r-1}{6 r(r+1)}\left(\sigma \frac{\partial E}{\partial z}-\nu E\right)_{i+1} \\
& +\frac{r^{2}+3 r+1}{6 r}\left(\sigma \frac{\partial E}{\partial z}-\nu E\right)_{i} \\
& +\frac{-r^{2}+r+1}{6(r+1)}\left(\sigma \frac{\partial E}{\partial z}-\nu E\right)_{i-1}
\end{aligned}
$$

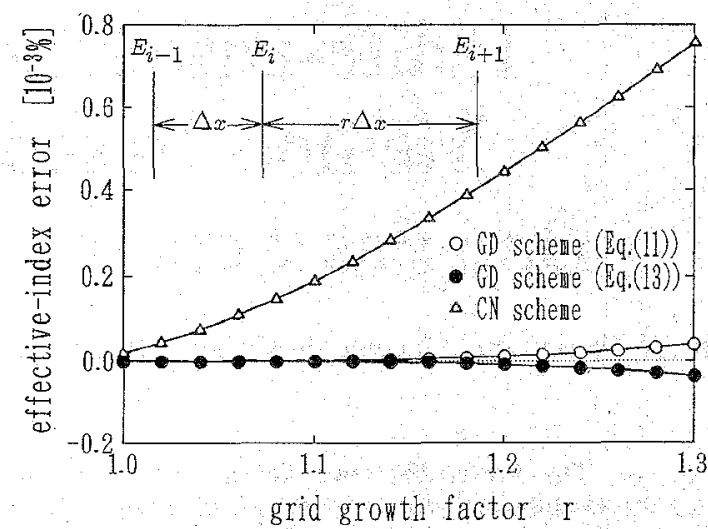

Fig. 2. Effective-index error as a function of grid growth factor $r$.

As can be expected, each of (11) and (13) is reduced to the uniform case when $r=1[7$, equation (3)]. It is obvious that each of (11) and (13) leads to a 6-point scheme, which can be efficiently solved by standard techniques. At the computational window edge, the transparent boundary condition [12] is easily incorporated.

\section{NUMERICAL RESULTS}

We treat a symmetric graded-index slab waveguide in which the refractive index is $n^{2}(x)=n_{s}^{2}+2 n_{s} \Delta n / \cosh ^{2}(2 x / w)$, where $n_{s}=2.1455, \Delta n=0.003$ and $w=5 \mu \mathrm{m}$. $\mathrm{A}$ wavelength considered here is $\lambda=1.3 \mu \mathrm{m}$. The lowest order eigenmode is analyzed using the imaginary-distance BPM [9]-[11].

We first summarize the results for a uniform grid $(r=1)$. The numerical parameters are as follows: the propagation step length $\Delta z=1 \mu \mathrm{m}$ and the total number of the transverse sampling points $2 M=400$. Fig. 1 shows the error in the effective index as a function of $\Delta x$. Also included for comparison is the result obtained with the conventional $\mathrm{CN}$ scheme. It should be noted that the error of the present scheme is almost proportional to $\Delta x^{4}$, while that of the conventional scheme is $\Delta x^{2}$

Since the reduced truncation error of the present scheme has been verified, consideration is next given to the case of a nonuniform grid. The grid spacing increases gradually from the center of the waveguide toward the edges of the computational window. Throughout this analysis, the grid growth factor $r$ is fixed. That is, the transverse sampling width is expressed as $\Delta x_{m}=r^{m-1} \Delta x_{1}, m=1, \cdots, M$. The initial sampling width is taken to be $\Delta x_{1}=0.208 \mu \mathrm{m}$. The calculation is made under the condition of almost the same computational window dimension of $2 L_{x} \simeq 83.2 \mu \mathrm{m}$. The propagation step length is $\Delta z=1 \mu \mathrm{m}$.

Fig. 2 shows the effective-index error as a function of grid growth factor $r$. In general, the accuracy gradually decreases as $r$ is increased. It should be noted, however, that the present scheme maintains high accuracy, even when $r$ is relatively large. In contrast, the accuracy for the conventional nonuniform $\mathrm{CN}$ scheme degrades considerably as $r$ is increased. It is interesting to note that (13) produces the error whose sign is opposite to that in (11). 


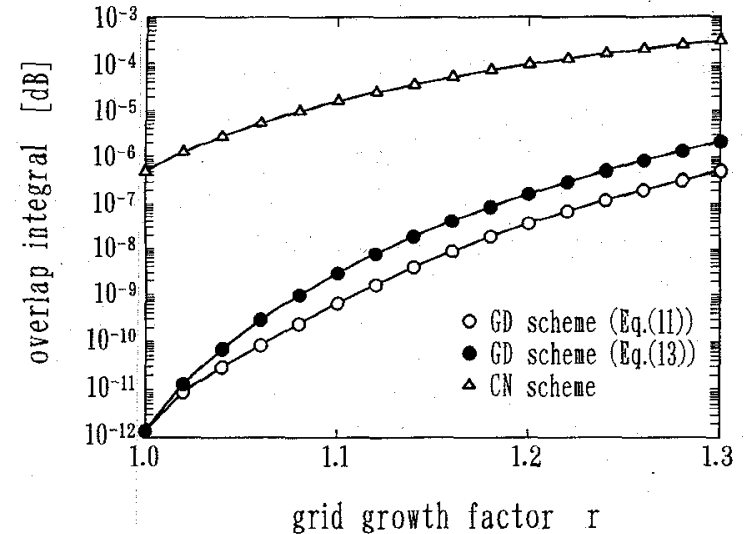

Fig. 3. Overlap integral between the numerically determined field and the exact solution as a function of grid growth factor $r$.

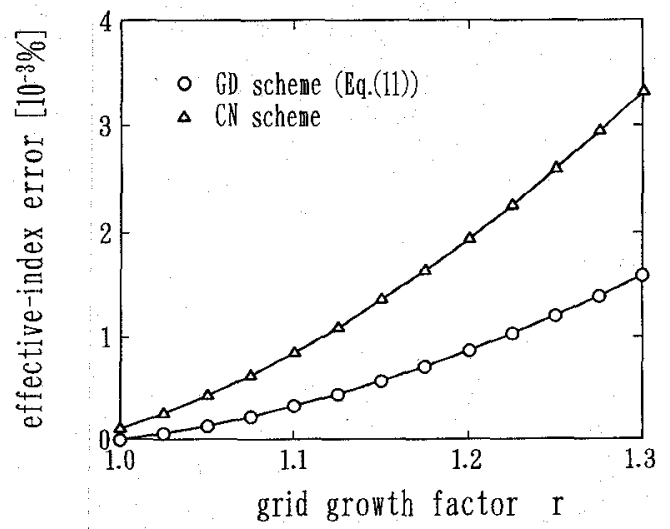

Fig. 4. Effective-index error of a three-dimensional waveguide as a function of grid growth factor $r$.

Consideration is also given to the accuracy of a field profile. The overlap integral between the numerical and exact solutions is evaluated. Fig. 3 shows that the overlap integral for the present scheme with $r=1.3$ nearly coincides with that for the conventional one with $r=1$. In the above analysis, the total number of the transverse sampling points $2 M$ are reduced from $400(r=1$ : a uniform grid) to 60,40 and 30 when $r$ is $1.1,1.2$ and 1.3 , respectively. This fact greatly contributes to reduction in computational time while maintaining its accuracy.

As a further application, we analyze a three-dimensional waveguide whose index profile takes a Gaussian function

$$
n(x, y)=n_{s}+\Delta n \exp \left(-x^{2} / d^{2}\right) \exp \left(-y^{2} / d^{2}\right)
$$

where $n_{s}=2.146, \Delta n=0.01$, and $d=3.5 \mu \mathrm{m}$. The computaional window size is approximately $2 L_{x} \times 2 L_{y}=16$ $\mu \mathrm{m} \times 16 \mu \mathrm{m}$. The initial sampling widths are $\Delta x_{1}=\Delta y_{1}=$ $0.2 \mu \mathrm{m}$, and $\Delta z=0.5 \mu \mathrm{m}, \lambda=1.3 \mu \mathrm{m}$. We apply (11) to the alternating-direction implicit method. Fig. 4 shows the effective-index error as a function of $r\left(=r_{x}=r_{y}\right)$, in which a value obtained from the GD scheme with $r=1$ is assumed to be exact. The GD scheme is also effective in the analysis of a three-dimensional waveguide.

The discussion in this letter has been restricted to the eigen mode analysis. When we apply the present scheme to the propagating beam analysis of a longitudinally varying structure, we may have to use an adaptive grid in which the missing values of the field are determined by an interpolation technique [13].

\section{CONCLUSION}

The generalized Douglas scheme for a nonuniform grid has been applied to the finite-difference beam propagation method. The formulation shows that the finite-difference equation results in a tridiagonal matrix achieving the truncation error of $O(\Delta x)^{4}$, provided the grid growth factor is $r=$ $1+O(\Delta x)$. The numerical results regarding a graded-index waveguide reveal the superiority of the present scheme over the conventional nonuniform scheme.

\section{ACKNOWLEDGMENT}

While the authors were preparing this manuscript, one of the authors (J. Yamauchi) had the opportunity to discuss a high accuracy scheme with Dr. G.R. Hadley of Sandia National Laboratories. The authors would like to express their gratitude to him for fruitful discussions.

\section{REFERENCES}

[1] D. Yevick, J. Yu, M. Munowitz, and D. Vezzetti, "Modal analysis of semiconductor rib waveguides employing nonequidistant grids," $J . O p t$. Soc. Amer. A, vol. 8, no. 9, pp. 1385-1388, 1991.

[2] Y. Chung, and N. Dagli, "Analysis of Z-invariant and Z-variant semiconductor tib waveguides by explicit finite difference beam propagation method with nonuniform mesh configuration," IEEE J. Quantum Electron., vol. 27, pp. 2296-2305, 1991

[3] T. Rasmussen, J. H. Povlsen, and A. Bjarklev, "Accurate finite difference beam propagation method for complex integrated optical structures," IEEE Photon. Technol. Lett., vol. 5, pp. 339-342, 1993.

[4] P. L. Liu, S. L. Yang, and D. M. Yuan, "The semivectorial beam propagation method;" IEEE J. Quantum Electron., vol. 29, pp. 1205-1211, 1993.

[5] G. S. Pu, T. Mizumoto, and Y. Naito, "Modified numerical technique for beam propagation method based on the Galerkin's technique," IEICE Trans. Electron., vol. E77-C, no. 3, pp. 510-514, 1994.

[6] D. Yevick, C. Rolland, W. Bardyszewski, and B. Hermansson, "Fresnel studies of reflected beams," IEEE Photon. Technol. Lett., vol. 2, pp. $490-492,1990$.

[7] J. Yamauchi, J. Shibayama, and H. Nakano, "Modified finite-difference beam propagation method based on the generalized Douglas scheme for variable coefficients," IEEE Photon. Technol. Lett., vol. 7, pp. 661-663, 1995.

[8] J. Yamauchi, O. Saito, and H. Nakano, "Improved finite-difference beam-propagation method for nonuniform grid," IEICE Spring Nat. Conv. Rec., C-211, 1995.

[9] D. Yevick, and W. Bardyszewski, "Correspondence of variational finitedifference (relaxation) and imaginary-distance propagation methods for modal analysis," Opt. Lett., vol. 17, no. 5, pp. 329-330, 1992.

[10] C. L. Xu, W. P. Huang, and S. K. Chaudhuri, "Efficient and accurate vector mode calculations by beam propagation method," J. Lightwave Technol., vol. 11, pp. 1209-1215, 1993.

[11] S. Jüngling and J. C. Chen, "A study and optimization of eigenmode calculations using the imaginary-distance beam-propagation method," IEEE J. Quantum Electron., vol. 30, pp. 2098-2105, 1994.

[12] G. R. Hadley, "Transparent boundary condition for beam propagation," Opt. Lett., vol. 16, no. 9, pp. 624-626, 1991.

[13] C. Massini, G. Bellanca, P. Bassi, and R. Sorrentino, "An FD-BPM with adaptive mesh for longitudially varying optical structure," Opt. Quantum Electron., vol. 27, no. 10, pp. 951-959, 1995. 